\title{
Corrigendum
}

\section{Expression of sphingosine I-phosphate receptor 4 and sphingosine kinase I is associated with outcome in oestrogen receptor negative breast cancer}

\section{J Ohotski, JS Long, C Orange, B Elsberger, E Mallon, J Doughty, S Pyne, NJ Pyne and J Edwards}

British Journal of Cancer (2012) I 07, 756. doi:I0.I038/bjc.2012.352 www.bjcancer.com

(c) 2012 Cancer Research UK

Correction to: British Journal of Cancer (2012) 106, 1453-1459. doi:10.1038/bjc.2012.98

In revision of the above paper during the proofing and correction process, an earlier version of Figure 3 was resupplied for publication and, subsequently, published in error. The authors and publishers apologise for this mistake.

The correct Figure 3 and associated legend are shown below.
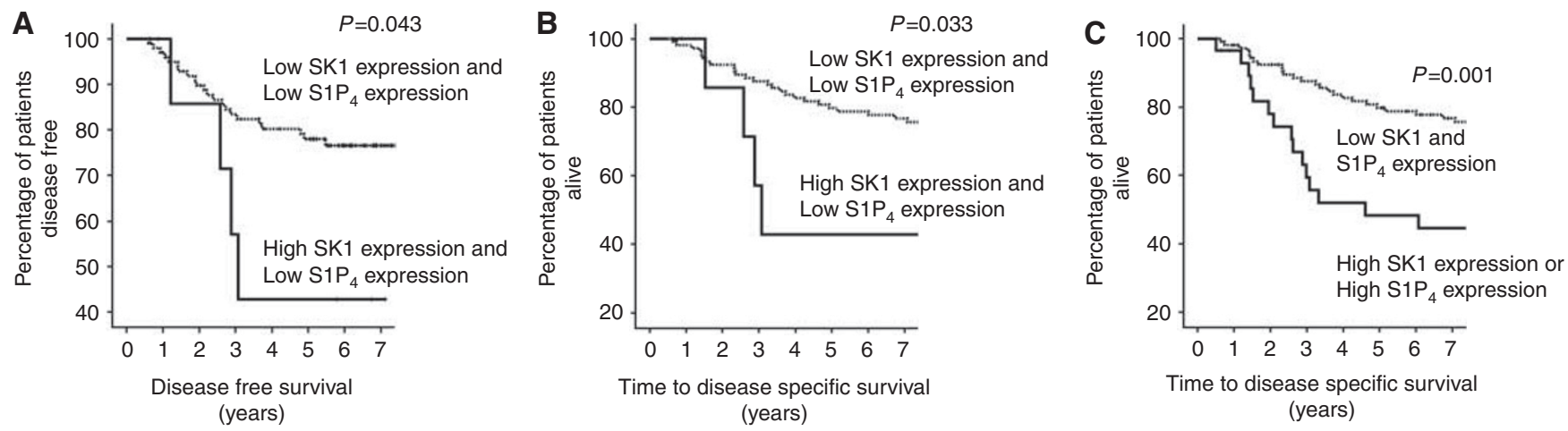

Figure 3 (A) High cytoplasmic SKI expression in a low SIP 4 expression background is associated with shorter disease-free survival compared with patients with low tumour $\mathrm{SIP}_{4}$ and SKI expression. (B) High cytoplasmic SKI expression in a low $\mathrm{SIP}_{4}$ expression background is associated with shorter disease-specific survival compared with patients with low tumour $\mathrm{SIP}_{4}$ and SKI expression. (C) High SKI or SIP $\mathrm{P}_{4}$ expression is associated with shorter disease-specific survival compared with patients with low SKI and $\mathrm{SIP}_{4}$ expression in their tumours. 